\title{
Kangaroo mother care for low birth weight babies: a randomized controlled trial in a tertiary care hospital of Bangladesh
}

\begin{abstract}
Background: $\mathrm{KMC}$ is a safe, low cost and effective method of caring for low birth-weight (LBW) babies to reduce neonatal morbidity and mortality.

Objectives: To assess the effect of KMC on LBW babies when compared with conventional method of care.

Methods: This randomized controlled trial study was conducted in neonatal ward of Dhaka Shishu (Children) Hospital, Dhaka, from November, 2014 to October 2015. All preterm LBW neonates with birth weight $1250 \mathrm{gm}$ to $1800 \mathrm{gm}$., gestational age $>30$ weeks to < 35 weeks, haemodynamically stable were given $\mathrm{KMC}$ and compared with conventional method of care. All the babies were monitored and data regarding weight, temperature, apnea, nosocomial infection, feeding, hospital stay and mortality etc. were recorded during the hospital stay and followed up to 40 weeks of gestational age. Statistic analysis was done by $\chi 2$, student $t$ test etc. with SPSS-17 version.
\end{abstract}

Results: comparison of $40 \mathrm{KMC}$ babies with 40 control showed that rate of weight gain per day in $\mathrm{KMC}$ group was $18.1 \pm 7.7 \mathrm{gms}$ and in control group, it was $13.0 \pm 4.5 \mathrm{gms}(\mathrm{p}<0.001)$. Apnea occurred less in KMC group ( $8 \%$ vs. $15 \%)(\mathrm{p}=0.22)$ and episodes of hypothermia was also recorded less in KMC group $(10 \%$ vs $18 \%))(p=0.21)$. Culture positive sepsis was $15 \%$ in $\mathrm{KMC}$ group and $20 \%$ in control group ( $\mathrm{p}=0.55$ The mean time to achieve full enteral feed in KMC group was $9.1 \pm 2.4$ days and $14.7 \pm 4,5$ days $(\mathrm{p}<0.001)$ in control group Ninety percent of $\mathrm{KMC}$ and $60 \%$ of control group babies were discharged with exclusive breast feeding ( $\mathrm{p}=0.002$ ). Mean hospital stay was $15.6 \pm 10.6$ days vs $18.2 \pm 4.5$ days, in $\mathrm{KMC}$ and control group respectively that was statistically insignificant, $\mathrm{p}=0.15$. The mortality was $2(5 \%)$ in KMC group and those was $6(15 \%)$ in control group and that was also statistically insignificant $(\mathrm{P}=0.14)$.

Conclusion: In this study, KMC is effective for caring of LBW babies and has better effect on weight gaining and establishment of exclusive breast feeding. So KMC is recommended for caring of low birth weight infants.

Keywords: kangaroo mother care; kmc; dhaka shishu hospital
Volume 7 Issue 2 - 2017

\section{Maksudur Rahman, MAK Azad Chowdhury, Md Mahbubul Hoque, Nishat Jahan, Liton Chandra Shaha}

Department of Neonatology, Bangladesh Institute of Child health(BICH), Dhaka Shishu (Children) Hospital, Bangladesh

\author{
Correspondence: Maksudur Rahman, Associate professor, \\ Department of neonatology, Bangladesh Institute of Child \\ health(BICH), Dhaka Shishu (Children) Hospital, Dhaka, \\ Bangladesh, Email maksuddr@gmail.com
}

Received: September 22, 2017 | Published: October II, 2017

\section{Introduction}

Bangladesh has one of the top 10 highest burdens of newborn deaths in the world. ${ }^{1}$ Nearly $45 \%$ of all newborn deaths are directly related to preterm birth and its complications. ${ }^{2}$ In conventional methods of care, Preterm and LBW (Low birth weight) babies need incubators which are costly, highly technical and need skilled personnel and proper maintenance. ${ }^{3}$ Moreover incubators separate babies from their mother, depriving them of the necessary contact. So good care of LBW babies is difficult, and hypothermia and nosocomial infections are frequent in low resource developing countries. Under such circumstances KMC (Kangaroo Mother Care) is a good alternative and some instance more effective than incubator in caring of preterm and LBW babies. To decrease neonatal morbidity and mortality, $\mathrm{KMC}$ is now considered as the most feasible, readily available, and preferred intervention in developed and developing countries. ${ }^{4}$ It is a special way of caring for the preterm or low birth weight infants where baby is kept in skin-toskin contact with the mother and breastfed exclusively. ${ }^{5}$. It is a safe and effective method of caring for low birth weight (LBW) infants, especially in low-resourced settings. ${ }^{4}$. It has also been described as a cost-effective, ${ }^{6,7}$ high-impact intervention ${ }^{8,9}$ for improving newborn survival. Beneficial physiological and behavioral effects of KMC for the infant has been well documented. Physiological effects include better thermoregulation, improved cardiorespiratory stability, lower risk of infection and faster growth. Behavioral effects relate to better sleep cycles, less crying and an analgesic effect during painful procedures. Beneficial effects for the mothers include better breastfeeding (increased milk production, exclusivity, duration, early initiation) and psychosocial effects (reduced anxiety, more maternal satisfaction, improved maternal-infant attachment and bonding). ${ }^{10,11}$ This study was conducted to assess the effect of KMC on LBW babies when compared with conventional method of care.

\section{Materials and methods}

This randomized controlled trial study was conducted in neonatal ward of Dhaka Shishu (Children) Hospital, Dhaka, Bangladesh from November, 2014 to October 2015. All preterm, neonates with birth weight $1250 \mathrm{gm}$ to $1800 \mathrm{gm}$., gestational age $>30$ weeks to < 35 weeks (Gestational age determined by New Ballard Score), haemodynamically stable (criteria of stable baby was 1) normal heart rate $100-160$ per minutes, 2) respiratory rate $30-59$ per minute with breaths comfortably, no sign of respiratory distress pink in room air or with $40 \%$ oxygen, no prolonged or frequent apnea) were included in this study. Babies having major life threatening congenital 
malformation, perinatal asphyxia and required ventilator or ion tropic support and babies with critically ill mother were excluded. Neonates in this study were randomly enrolled by inclusions and exclusions criteria, and were allocated either KMC group or control group by simple randomization with concealed sealed envelope technique. Forty neonates (40) were in KMC group and forty neonates (40) were in control group. Neonates in control group were managed under radiant warmer. A written informed consent was obtained from all mothers. Details of antenatal period and delivery were recorded. During hospital stay, both the groups were monitored for daily weight gain, episodes of hypothermia, apnea, nosocomial infection, physiological parameters (heart rate, respiratory rate, axillary temperature and oxygen saturation). Data regarding feeding was also recorded. After discharge, the neonates were followed weekly till 40 weeks of corrected gestational age. All information was recorded according to follow up sheet. In KMC group mother or caregiver was explained detail about KMC adoption in presence of their family. $\mathrm{KMC}$ was initiated as soon as the baby was stable. If the mother was not available, KMC was started with the help of any other family member. In KMC position, baby was kept skin to skin contact with mother bare chest in between the breasts. Baby was remained froglike position, head turned in one side with neck slight extended, wearing front open sleeveless shirt, cap, shocks and a nappy. There was a binder for support the baby around baby and mother. Comfortable bed or chair was provided to the mother or caregiver practicing $\mathrm{KMC}$ in ward. KMC was given at least 2 hours at a time and at least 12 hours in a day. When the baby was not in $\mathrm{KMC}$, the baby placed in cot with adequate cloths and coverings. Infant in both the groups were discharged when following criteria were achieved: Baby's general health was good, no evidence of infection and no I/V medications. Baby was feeding well and receiving breast milk directly or by cup and spoon gaining weight (at least $15 \mathrm{gm} / \mathrm{kg} /$ day) for 3 consecutive days. Maintaining body temperature well without assistance for at least 3 consecutive days and Mother and family members were confident to care the baby. Besides KMC and radiant warmer care, babies of both groups were treated with conventional hospital protocol. Data were analyzed by chi-square $\left(\chi^{2}\right)$ test, unpaired student's ' $t$ ' test with the help of SPSS version-17.

\section{Calculation of sample size}

$$
\begin{aligned}
& n=\frac{P_{1}\left(1-P_{1}\right)+P_{2}\left(1-P_{2}\right) \times\left(Z_{\infty}+Z_{\beta}\right)^{2}}{\left(P_{1}-P_{2}\right)^{2}} \\
& =76(100-76)+98(100-98) \times(1.96+0.85)^{2} \\
& \quad(76-98)^{2}
\end{aligned}
$$

[Here

$P_{1}=$ Outcome in control $76 \%[12]$

$P_{2}=$ Outcome in case $98 \%$

$$
Z_{\infty}=1.96
$$$$
\left.\left.Z_{\beta}=0.85 \text { (at } 80 \% \text { power }\right)\right]
$$$$
=32(\text { Approx }) \text { in each groups }
$$

\section{Results}

In this study, comparison of $40 \mathrm{KMC}$ babies with 40 control showed that rate of weight gain per day in KMC group was 18.1 \pm 7.7 gms and in control group, it was $13.0 \pm 4.5$ gms $(\mathrm{p}<0.001)$. Apnea occurred less in KMC group ( $8 \%$ vs. $15 \%)(\mathrm{p}=0.23)$ and episodes of hypothermia was also recorded less in KMC group (10\% vs $18 \%)$ ) $(\mathrm{p}=0.21)$. But both are statistically insignificant. Culture positive sepsis was $15 \%$ in $\mathrm{KMC}$ group and $20 \%$ in control group $(\mathrm{p}=0.56)$. The mean time to achieve full enteral feed in KMC group was 9.1 \pm 2.4 days and $14.7 \pm 4.5$ days $(p<0.001)$ in control group. Ninety percent of $\mathrm{KMC}$ and $60 \%$ of control group babies were discharged with exclusive breast feeding $(\mathrm{p}=0.002)$. The rest received cup feeding along with the breast feeding. In this study mean hospital stay was $15.6 \pm 10.6$ days vs $18.2 \pm 4.5$ days, in $\mathrm{KMC}$ and control group respectively that was statistically insignificant, $\mathrm{p}=0.15$. The mortality was $2(5 \%)$ in $\mathrm{KMC}$ group and those was $6(15 \%)$ in control group and that was also statistically insignificant $(\mathrm{P}=0.14)$ (Tables 1-4).

\section{Discussion}

This study was conducted at Dhaka shishu (children) hospital from November, 2014 to October, 2015. In this period, 40 KMC babies were compared with same number of babies who did not receive $\mathrm{KMC}$. Although $\mathrm{KMC}$ is a key intervention package in newborn health initiatives, there are several barriers to $\mathrm{KMC}$ practice that mothers and other stakeholders face while practicing KMC. These barriers can exist at multiple levels, including barriers to implementation of a KMC program, deficiencies in the program itself, or specific challenges associated with the practice of KMC which the mother has to perform. ${ }^{13}$. To overcome barriers, there are also positive factors that can enable a mother to practice KMC. In spite of benefits of KMC, mothers may face barriers to practice this, some of which may prevent them from achieving the continuous STS contact with their infants (a defining feature of KMC). In India 6.5\% mother felt that KMC could be given 12 hour/day or greater and $52 \%$ mother felt that KMC could be given practically 1 hour /d. In another study, only $23.8 \%$ mother practiced STS (skin to skin contact) for more than 7 hours / day in the first 48 hours of life, and the average number of hours of STS during days $3-7$ of life was $2.7 \pm 3.4$ hours. ${ }^{13,14}$

In one systemic review shows that the top barriers to KMC practice are: "Issues with the facility environment / resources," "Negative impressions of staff attitudes or interactions with staff," "lack of help with KMC practice or other obligations," and "low awareness of KMC / infant health." On the other hand, the enablers of KMC practice are "Mother-infant attachment," "Feelings of confidence / empowerment," and "Ease of practice / preference over traditional care", "Support from family, friends, and other mothers," "Support from staff or community health worker (CHW) and "Understanding of efficacy" etc. ${ }^{13}$ In this study though it was not analyzed statistically few barriers were observed regarding practicing of KMC. The main barrier was absent of appropriate helper of mother that means any person, nurse or family member who helps mother during KMC practice, during her discomfort and bathing etc. Without a continuous presence of a helper KMC cannot be continued. Unwillingness of practicing $\mathrm{KMC}$, that was overcome with repeated counseling, absence of proper monitoring of KMC practice at home, absence of appropriate privacy of mother, sweating of mother specially during summer season were important barriers of KMC practice in this study.

Low awareness of KMC and infant health more broadly were important barriers to $\mathrm{KMC}$ practice seen in majority of publications. 
But studies done in India, Ghana, Bangladesh showed that mother who were trained, practicing $\mathrm{KMC}$ or got massage about $\mathrm{KMC}$, could understand about KMC and knew the benefits of KMC. ${ }^{15-17}$ Several studies in South Africa and Ghana, showed that mothers belief in the benefit of KMC, improvement of heath of child by practicing KMC and hearing the success story about $\mathrm{KMC}$ were the main motivation to continue KMC. ${ }^{18,19}$

Table I Distribution of the patients according to particulars of the patients of comparison groups

\begin{tabular}{llll}
\hline Variables & Case $(\mathbf{n = 4 0 )}$ & Control $(\mathbf{n = 4 0 )}$ & p value \\
\hline Age at admission (day)(Mean SD) & $1.8 \pm I . I$ & $2.1 \pm 1.2$ & 0.24 \\
Sex of the baby & No. $(\%)$ & No. $(\%)$ & \\
Male & $24(60 \%)$ & $28(70 \%)$ & 0.35 \\
Female & $16(40 \%)$ & $12(30 \%)$ & \\
\hline
\end{tabular}

Table 2 Feeding outcome between KMC and conventional care group

\begin{tabular}{|c|c|c|c|}
\hline Hospital course & $\begin{array}{l}\text { Case } \\
(n=40) \\
\text { No. }(\%)\end{array}$ & $\begin{array}{l}\text { Control } \\
(n=40) \\
\text { No. }(\%)\end{array}$ & p value \\
\hline \multicolumn{4}{|l|}{ Start of first feed on } \\
\hline Ist day & $13(33.0 \%)$ & $8(20.0 \%)$ & \multirow{3}{*}{$0.175 \mathrm{~ns}$} \\
\hline 2-3 days & $20(50.0 \%)$ & $19(48.0 \%)$ & \\
\hline 4-5 days & $7(17.0 \%)$ & $13(32.0 \%)$ & \\
\hline Time of achieve full enteral feeding in days & $9.08 \pm 2.35$ & $14.735 \pm 4.54$ & $<0.001 *$ \\
\hline Exclusive breast feeding & $36(90.0 \%)$ & $24(60.0 \%)$ & \multirow[b]{2}{*}{$<0.001^{*}$} \\
\hline Yes & $4(10.0 \%)$ & $16(40.0 \%)$ & \\
\hline
\end{tabular}

Statistical analysis was done by Chi-square test and unpaired' test.

$P<0.05$ was taken as statistically significant,

$\mathrm{n}=$ Total number of subjects

Table 3 Showing rate of weight gain between KMC and conventional care group

\begin{tabular}{|c|c|c|c|}
\hline Variables & $\begin{array}{l}\text { Case } \\
(n=40) \\
\text { Mean } \pm S D\end{array}$ & $\begin{array}{l}\text { Control } \\
(n=40) \\
\text { Mean } \pm S D\end{array}$ & $p$ value \\
\hline Weight gain started(days) & $5.9 \pm 1.8$ & $8.6 \pm 2.3$ & $<0.00 I^{*}$ \\
\hline Birth weight regained (days) & $10.8 \pm 2.9$ & $13.2 \pm 3.8$ & $<0.00 I^{*}$ \\
\hline Rate of weight gain (gms) & $18.1 \pm 7.7$ & $13.0 \pm 4.5$ & $<0.00 I^{*}$ \\
\hline
\end{tabular}

Statistical analysis was done by unpaired' test.

$\mathrm{P}<0.05$ was taken as statistically significant, $\mathrm{n}=$ Total number of subjects

Table 4 Distribution of the patients according to hospital course of the patients

\begin{tabular}{|c|c|c|c|}
\hline Hospital course & $\begin{array}{l}\text { Case } \\
(n=40) \\
\text { No. (\%) }\end{array}$ & $\begin{array}{l}\text { Control } \\
(n=40) \\
\text { No. }(\%)\end{array}$ & p value \\
\hline $\begin{array}{l}\text { Episode of hypothermia } \\
\text { Yes } \\
\text { No }\end{array}$ & $\begin{array}{l}4(10 \%) \\
36(90 \%)\end{array}$ & $\begin{array}{l}7(18 \%) \\
33(82 \%)\end{array}$ & 0.21 \\
\hline $\begin{array}{l}\text { Episode of apnea } \\
\text { Yes } \\
\text { No }\end{array}$ & $\begin{array}{l}3(7.5 \%) \\
37(92.5 \%)\end{array}$ & $\begin{array}{l}6(15 \%) \\
34(85 \%)\end{array}$ & 0.23 \\
\hline $\begin{array}{l}\text { Culture proven sepsis } \\
\text { Yes } \\
\text { No }\end{array}$ & $\begin{array}{l}6(15.0 \%) \\
34(85.0 \%)\end{array}$ & $\begin{array}{l}8(20.0 \%) \\
32(80.0 \%)\end{array}$ & 0.56 \\
\hline $\begin{array}{l}\text { Exclusive breast feeding } \\
\text { Yes } \\
\text { No }\end{array}$ & $\begin{array}{l}36(90.0 \%) \\
4(10 \%)\end{array}$ & $\begin{array}{l}24(60.0 \%) \\
16(40.0 \%)\end{array}$ & $0.002 *$ \\
\hline $\begin{array}{l}\text { Mortality } \\
\text { yes } \\
\text { No }\end{array}$ & $\begin{array}{l}2(5 \%) \\
38(95 \%)\end{array}$ & $\begin{array}{l}6(15 \%) \\
34(85 \%)\end{array}$ & 0.14 \\
\hline Mean hospital stay (days: mean $\pm \mathrm{SD}$ ) & $15.6 \pm 10.6$ & $18.2 \pm 4.5$ & 0.15 \\
\hline
\end{tabular}

Statistical analysis was done by Chi-square test and unpaired' test.

$\mathrm{P}<0.05$ was taken as statistically significant, $\mathrm{n}=$ Total number of subjects 
KMC also limits the mother's ability to take care of other tasks and obligations. "Lack of help with KMC practice and other obligations" were the barriers to continue KMC. But "Support from family, friends, and other mothers" were the enabler to practice KMC. One study in Malawi, showed that the involvement of the grandma during the admission was the most effective way to ensure KMC continues at home. Similarly critical roles of family members providing emotional support were documented in Ghana and South Africa. ${ }^{19,20}$

Resourcing and sociocultural factors emerged as the top barriers to $\mathrm{KMC}$ adoption for nurses. In case of resourcing, "actual increased workload / staff shortages" and "Lack of clear guidelines / training" were the main barriers. Whereas regarding sociocultural barriers, "General lack of buy-in / belief in efficacy" and "Concerns about other medical conditions / care" were also in the top five barriers for nurses..$^{13}$

In case of barriers for fathers, CHW's, physicians, and program managers, "Lack of opportunity to practice," "General lack of buyin / belief in efficacy and "Need for high-touch support from staff." were the main barriers for KMC practice respectively. So by proper identifying the barriers and enablers to practice $\mathrm{KMC}$ are the main key factors for successful implementation of KMC. ${ }^{13}$ As with any other intervention KMC has certain costs too. The cost of KMC practice varies according to implementation cost, paying -nonpaying beds cost, salary structure of staffs, logistic cost etc. In this study for implementation of KMC in existing neonatology ward, it was needed bed, chair with back support, baby's clothes, cap, socks, nappy, mother's gown, binder which cost about 122.25US\$ / baby and incubator cost about 5000 US\$/baby plus maintenance cost. The cost of 2 days training program for health personnel about KMC initially, was about 375 US\$. The less hospital stay also lessened the cost of KMC. Edward I.et al did a study to examine the costs of implementing kangaroo mother care (KMC) in a referral hospital in Nicaragua, including training, implementation, and ongoing operating costs, and to estimate the economic impact on the Nicaraguan health system and compared before and after the implementation. In this study the intervention cost was US\$ 23113 but the money saved with shorter hospitalization, elimination of incubator use, and lower antibiotic and infant formula costs made up for this expense in 1-2 months. Extending KMC to 12 other facilities in Nicaragua that was projected to save approximately US\$166 000 (based on the referral hospital incubator use estimate) or US\$233000 after one year. ${ }^{21}$. Cattaneo et al. evaluated the cost efficiency of KMC when compared to conventional methods of care (CMC). The cost variables included two types of running costs: salaries and other items (food for mothers and babies, laundry and linen, drugs and other medical supplies, X-ray and laboratory, fuel and electricity and maintenance of equipment. The authors showed that KMC was cheaper than conventional methods of care (CMC) in terms of salaries (US\$ 11,788 vs US\$29,888) and other running costs (US\$ 7501 vs US\$ 9876). ${ }^{22}$

Reza Gholi Vahidi et al. showed in their study that though KMC's unit cost was a little higher than Conventional method, but comparing its positive outcomes on breastfeeding's and mortality it was considered as cost effective method..$^{23}$ Deepak Sharma et al. in India showed that there was significant saving of around 33800 INR (USD) in the $\mathrm{KMC}$ group for each patient. ${ }^{24}$ Jannati et al. in India conducted a retrospective cost effective analysis of $\mathrm{KMC}$ and $\mathrm{CMC}$ and showed that the cost savings was nearly $630 \$$ per patient. So KMC is cost effective intervention. ${ }^{23}$

A global health recommendation is that infants should be exclusively breast fed for the first six months of life as because breast milk mediates unequalled beneficial effects regarding nutritional, immunological, and cognitive outcomes. ${ }^{25}$ Managing these preterm babies are clinically more challenging in initiation and sustainability of breastfeeding as their suckling behavior is not mature and requires neonatal care for a sustainable period. In Bangladesh exclusive breastfeeding rate has declined from 62 to 54 and there is no data on preterm breastfeeding rate. As preterm rate is high in Bangladesh it might have influence on declining exclusive breastfeeding rate. ${ }^{26}$ A study showed that among Canadian babies weighing less than $2500 \mathrm{gm}$ only $58 \%$ had been breastfed at birth, compared to $73 \%$ in term group. Among LBW infants only 3\% were discharged on exclusive breastfeeding. ${ }^{27}$ Study done in Malaysia showed, only $40.2 \%$ of VLBW babies were being breastfed at the time of discharge from hospital despite breastfeeding incentive programs adopted by the hospital. ${ }^{28}$ In this study we have found that early feeding could have been started in LBW and VLBW babies who were managed in $\mathrm{KMC}$ and also the time of achieving full enteral feeding was earlier and rate of exclusive breastfeeding $(90 \%$ vs. $60 \%)$ was found higher in these babies. A study from Sweden done by Whitelaw et al found that 71 preterm babies weighing less than $1500 \mathrm{~g}$ submitted to KMC had a two time higher prevalence of breast feeding than the control group at six weeks of life (55\% versus 28\%). ${ }^{29}$ Kangaroo Mother Care (KMC), which has an extensive research base, is regarded as a successful way to empower mothers to become familiar with their infants, strengthens their mothering at their own pace, and increases breastfeeding rate and duration. ${ }^{30,31}$

As it is known that neonate usually loses weight in his first week of life than starts gaining weight, similar trend was seen in this study. But in this study babies with KMC had early gaining weight and early regaining weight than babies with conventional care $(p<.001)$. Moreover weight gain was significantly higher in KMC babies than nonKMC babies $(\mathrm{p}<001)$. Similarly in another study, it was found that $\mathrm{KMC}$ babies achieved significantly better growth (KMC; $24.0 \mathrm{gms}$ vs Conventional Care; $15.6 \mathrm{gms}, \mathrm{p}<0.001 .{ }^{32}$ Meta analysis of KMC studies confirmed greater wt gain in KMC infants than in infants not managed in $\mathrm{KMC}^{33}$

In this study apnea occurred in $8 \%$ babies in KMC group and 15\% cases in control group which was statistically insignificant $(\mathrm{p}=0.23)$. In the study of Chowdhury et al. apnea in KMC group was found less (KMC: $2,8 \%$ vs Control: $8,32 \%, \mathrm{p}=0.03) .{ }^{34}$ The episodes of hypothermia was $10 \%$ in $\mathrm{KMC}$ group and $18 \%$ in control group that was also statistically insignificant $(\mathrm{p}=0.21)$ in this study. Several studies showed that incidence of hypothermia were less in KMC group. Cattaneo et al showed in their study that hypothermia was more in babies that did not get KMC (31.5 vs 13.5 episode/100 infants). ${ }^{35}$

There were no statistical differences between two groups regarding culture positive sepsis. It was found $15 \%$ cases in KMC group and $20 \%$ babies in control group $(\mathrm{p}=0.56)$. But Chowdhury et al showed culture positive sepsis was more in babies who did not get KMC than who got KMC..$^{34}$

In this study mean hospital stay was $15.6 \pm 10.6$ days vs $18.2 \pm$ 4.5 days in $\mathrm{KMC}$ and control group respectively that was statistically insignificant $(\mathrm{p}=0.15)$. There was no difference in hospital stay between $\mathrm{KMC}$ and no-KMC group in the study done by Rao et al (KMC vs no-KMC: 12.8 days vs 12.9 days, $\mathrm{P}=0.93$ ). ${ }^{32}$ But Chowdhury et al. found less hospital stay in KMC group in their study and which was statistically significant. ${ }^{34}$

Mortality was $2(5 \%)$ in $\mathrm{KMC}$ group and those was $6(15 \%)$ in control group and that was statistically insignificant, $\mathrm{P}=0.14$. But meta-analysis of three RCTs showed a significant reduction in 
neonatal mortality[relative risk (RR) $0.49,95 \%$ confidence interval (CI) 0.29-0.82] compared with standard care. ${ }^{35}$

This study is novel as preterm baby is more in this country and place of this study is a tertiary care hospital in Bangladesh where most of the preterm babies are referred from other districts. But there are short of costly incubator in this hospital as well as others hospitals. By conducting such type of study in this hospital further substantiate the efficacy of KMC and motivates the other health personnel to practice this simple, effective, low cost technique in their resource poor health facilities. So morbidity and mortality of neonates would be reduced.

\section{Conclusion}

In this study, KMC is effective for caring of LBW babies and has better effect on weight gaining and establishment of exclusive breast feeding. So KMC is recommended for caring of low birth weight infants.

\section{Acknowledgments}

Professor (Dr.) MAK Azad Chowdhury,Member of Technical committee of KMC, Bangladesh Head of KMC Project, Dhaka Shishu (Children) Hospital, Dhaka. Prof. and head, Department of neonatology \& Academic director.

Bangladesh Institute of Child Health (BICH), Dhaka Shishu (Children) Hospital, Dhaka, Bangladesh.

\section{Conflicts of interest}

None.

\section{References}

1. Lawn JE, Cousens S, Zupan J. 4 million neonatal deaths:When? Where? Why?Lancet 365(9462):891-900

2. Ahmed FA. UNICEF Saving preterm babies in Bangladesh hospitals. 2012. 2005

3. Mohammadzadeh A, Farhat A, Jafarzadeh M, et al. Advantages of kangaroo mother care in less than 2000 grams low birth weight neonates. M JIRI. 2011;25(1):11-15.

4. Bergh AM, Manu R, Davy K, et al. Progress with the implementation of kangaroo mother care in four regions in Ghana. Ghana Med J. 2013;47(2):57-63 .

5. World health organization. Kangaroo Mother Care:a practical guide. Department of reproductive Health and research, WHO, Geneva, Switzerland, 2003. p. 1-54.

6. Darmstadt GL, Bhutta ZA, Cousens S, et al. Evidence-based, cost effective interventions:how many newborn babies can we save? Lancet. 2005;365(9463):977-988

7. Victora CG, Rubens CE, GAPPS Review Group. Global report on preterm birth and stillbirth (4 of 7):delivery of interventions. $B M C$ Pregnancy Childbirth. 2010;10(S1):S4.

8. Lawn JE, Kerber K, Enweronu LC, et al. (2010) 3.6 Million neonatal deaths-what is progressing and what is not? Semin Perinatol. 2010;34(6):371-386.

9. Barros FC, Bhutta ZA, Batra M, et al. Global report on preterm birth and stillbirth (3 of 7):evidence for effectiveness of interventions. $B M C$ Pregnancy and Childbirth.12010;0(S1):S3.

10. Ludington HSM, Morgan K, Abouelfettoh A. A clinical guideline for implementation of kangaroo care with premature infants of 30 or more weeks' postmenstrual age. Adv Neonatal Care. 2008;8(S3):S3-S23.
11. Ruiz JG, Charpak N. Evidence-based clinical guidelines for an optimal use of the kangaroo mother method in preterm and/or low birth weight infants at birth. Bogotá, Colombia:Fundación Canguro and Department of Clinical Epidemiology and Biostatistics, School of Medicine, Pontificia Universidad Javeriana. 2007.

12. Rao SPN, Udani R, Navavati R. Kangaroo mother care for low birth weight infants:a randomized controlled trial. Indian pediatrics. 2008;45(1):17-23.

13. Muddu GK, Boju SL, Chodavarapu R. Knowledge and Awareness about Benefits of Kangaroo Mother Care. Indian J Pediatr. 2013;80(10):799803

14. Parmar VR, Kumar A, Kaur R, et al. Experience with kangaroo mother care in a neonatal intensive care unit (NICU) in Chandigarh, India. Indian J Pediatr. 2009;76(1):25-28.

15. Quasem I, Sloan NL, Chowdhury A, et al. Adaptation of kangaroo mother care for community-based application. J Perinatol. 2003;23(8):646651

16. Bergh AM, Manu R, Davy K, et al. Translating research findings into practice - the implementation of kangaroo mother care in Ghana. Implement Sci. 2012;7:75.

17. Victor L, Persoon J. Implementation of kangaroo care:a parent-health care team approach to practice change. Crit Care Nurs Clin North Am. 1994;6(4):891-895.

18. Bazzano A, Hill Z, Tawiah-Agyemang $\mathrm{C}$, et al. Introducing home based skin-to-skin care for low birth weight newborns:a pilot approach to education and counseling in Ghana. Global health promotion. 2012;19:42-49.

19. Heinemann AB, Hellstrom-Westas L, Hedberg Nyqvist K. Factors affecting parents' presence with their extremely preterm infants in a neonatal intensive care room. Acta Paediatr. 2013;102(7):695-702.

20. Broughton EI, Gomez I, Sanchez N, et al. The cost-savings of implementing kangaroo mother care in Nicaragua. Rev Panam Salud Publica. 2013;34(3):176-182.

21. Cattaneo A, Davanzo R, Worku B, et al. Kangaroo mother care for low birthweight infants:a randomized controlled trial in different settings. Acta Paediatr Oslo Nor. 1998;87(9):976-985.

22. Vahidi RG, Gholipour K, Jannati A, et al. Cost and Effectiveness Analysis of Kangaroo Mother Care and Conventional Care Method in Low Birth Weight Neonates in Tabriz 2010-2011. Journal of Clinical Neonatology. 2014;3(3):148-152.

23. Sharma D, Murki S, Oleti TP. To compare cost effectiveness of 'Kangaroo Ward Care' with 'Intermediate intensive care' in stable very low birth weight infants (birth weight $<1100$ grams):a randomized control trial. Ital J Pediat. 2016; 42:64.

24. Seidman G, Unnikrishnan S, Kenny E, et al. Barriers and Enablers of Kangaroo Mother Care Practice:A Systematic Review. PLoS ONE. 2015;10(5):e0125643.

25. Kramer MS, Kakuma R. The optimal duration of exclusive breastfeeding. A systemic review. Adv Exp Med Biol. 2002;554:63-77.

26. Bangladesh Demographic Health Survey (BDHS), National Institute of Population Research and Training Ministry of Health and Family Welfare Dhaka, Bangladesh, 2014. pp. 1-354.

27. Blencowe H, Cousens S, Oestergaard MZ, et al. National, regional and worldwide estimates of preterm birth rates in the year 2010 with time trends since 1990 for selected countries:a systematic analysis and implications. Lancet. 2012;379(9832):2161-2172.

28. Lefebvre F, Ducarme M. Incidence and duration of lactational performance among mother of low-birth-weight infants. CMAJ. 1989;140(10):1159-1164 
29. Boo NY, Goh ES. Predictors of breast feeding in very low birth weight infants at the time of discharge from hospital. J Trop Pediatr. 1999;45(4):195-201.

30. Feldman R, Eidelman AI, Sirota L, et al. Comparison of skin-to-skin (Kangaroo) and traditional care:Parenting outcome and preterm infant development. Pediatrics. 2002;110(1-1):16-26.

31. Hake-Brooks SJ, Anderson GC. Kangaroo care and breastfeeding of mother-preterm infant dyads 0-18 months:A randomized control trail. Neonatal Netw. 2008;27(3):151-159.

32. Rao PN, Udani R, Nanavati R. Kangaroo Mother Care for Low birth weight infants:a randomized controlled trial. Indian pediatr 2008;45(1):17-23.
33. Conde-Agudelo A, Diaz Rosselo JL, Belizan JM. Kangaroo Morther Care to reduce mortality and morbidity in low birth weight infants. Cochrane Database Syst Rev. 2004;22(4):CD002771.

34. Chowdhury RM. Kangaroo Mother Care as compared to standard care for management of preterm low birth weight babies. MD Thesis, BSMMU, Dhaka. 2013.

35. Rekha HU, Nandkishor K, Ruchi NN, et al. Kangaroo mother care:A cohort follow up study on impact of duration of KMC on mortality, morbidity, hospital stay and breastfeeding. Journal of Neonatology. 2013;27(1):5-10. 